\title{
Constraining Cosmological Parameters Based on Relative Galaxy Ages
}

\section{Citation}

Jimenez, Raul, and Abraham Loeb. 2002. “Constraining Cosmological Parameters Based on Relative Galaxy Ages." The Astrophysical Journal 573 (1): 37-42. https://doi.org/10.1086/340549.

\section{Permanent link}

http://nrs.harvard.edu/urn-3:HUL.InstRepos:41393308

\section{Terms of Use}

This article was downloaded from Harvard University's DASH repository, and is made available under the terms and conditions applicable to Other Posted Material, as set forth at http:// nrs.harvard.edu/urn-3:HUL.InstRepos:dash.current.terms-of-use\#LAA

\section{Share Your Story}

The Harvard community has made this article openly available.

Please share how this access benefits you. Submit a story.

\section{Accessibility}




\title{
CONSTRAINING COSMOLOGICAL PARAMETERS BASED ON RELATIVE GALAXY AGES
}

\author{
RAUl JimEnez ${ }^{1}$ AND ABRAHAM Loeb ${ }^{2}$ \\ Received 2001 June 7; accepted 2002 March 7
}

\begin{abstract}
We propose to use relative galaxy ages as a means of constraining cosmological parameters. By measuring the age difference between two ensembles of passively evolving galaxies at somewhat different redshifts, one could determine the derivative of redshift with respect to cosmic time, $d z / d t$. At high redshifts, $z \sim 1-2$, this measurement would constrain the equation of state of the dark energy, while at low redshifts, $z \lesssim 0.2$, it would determine the Hubble constant, $H_{0}$. Furthermore, we show that $d^{2} z / d t^{2}$ directly tracks the equation of state of the dark energy; thus, measurements of this quantity at low redshifts can be used to constrain it. The selected galaxies need to be passively evolving on a time much longer than their age difference.
\end{abstract}

Subject headings: cosmology: theory — galaxies: stellar content

\section{INTRODUCTION}

Recently, there has been much progress in constraining cosmological parameters such as the matter content of the universe (Peacock et al. 2001) and the Hubble constant (Freedman et al. 2001). Available data for the microwave background anisotropies on degree scales (de Bernardis et al. 2000; Hanany et al. 2000; Netterfield et al. 2002; Lee et al. 2001) and the Hubble diagram of Type Ia supernovae (Riess et al. 1998; Perlmutter et al. 1999) indicate that the universe has a flat geometry and is dominated at present by some form of dark energy with a negative pressure (Garnavich et al. 1998; Perlmutter et al. 1999). The equation of state of the dark energy, $p_{Q}=w_{Q} \rho_{Q}$, expresses the ratio between the pressure, $p_{Q}$, and the mass density, $\rho_{Q}$, of the dark energy in terms of the parameter $w_{Q}$ (in units of $c=1$ ). The value of $w_{Q}$ could either be constant, as in the case of a cosmological constant $\left(w_{Q}=-1\right)$, or be time-dependent, as in the case of a rolling scalar field or "quintessence" (Ratra \& Peebles 1988; Caldwell, Dave, \& Steinhardt 1998). Any such behavior would have far-reaching implications for particle physics. Therefore, the next observational challenge is to determine the evolution of $w_{Q}$ as a function of redshift (Huterer \& Turner 2001; Maor, Brustein, \& Steinhardt 2001; Weller \& Albrecht 2001). The related observations need to be done at redshifts $z \lesssim 2$, when the dark energy started to dominate the expansion of the universe.

The popular approach for measuring $w_{Q}(z)$ uses its effect on the luminosity distance of sources. In particular, the proposal for the Supernova/Acceleration Probe (SNAP) mission $^{3}$ suggests monitoring $\lesssim 2000$ Type Ia supernovae across the sky per year and determining their luminosity distances up to a redshift $z \sim 1.5$ with high precision. However, the sensitivity of the luminosity distance to the redshift history of $w_{Q}(z)$ is compromised by its integral nature (Maor et al. 2001),

$$
d_{L}=(1+z) \int_{z}^{0}\left(1+z^{\prime}\right) \frac{d t}{d z^{\prime}} d z^{\prime},
$$

${ }^{1}$ Department of Physics and Astronomy, Rutgers University, 136 Frelinghuysen Road, Piscataway, NJ 08854-8019;

raulj@physics.rutgers.edu.

2 Astronomy Department, Harvard University, 60 Garden Street, Cambridge, MA 02138; aloeb@cfa.harvard.edu.

${ }^{3}$ SNAP mission proposal information is available at http://snap.lbl.gov/. where $t(z)$ is the age of the universe at a redshift $z$, which depends on $w_{Q}(z)$.

In this paper, we propose an alternative method that offers a much better sensitivity to $w_{Q}(z)$ because it measures the integrand of equation (1) directly. Any such method must rely on a clock that dates the variation in the age of the universe with redshift. The clock in our method is provided by spectroscopic dating of galaxy ages. Based on measurements of the age difference, $\Delta t$, between two passively evolving galaxies that formed at the same time but are separated by a small redshift interval $\Delta z$, one can infer the derivative $(d z / d t)$ from the ratio $(\Delta z / \Delta t)$. The statistical significance of the measurement can be improved by selecting fair samples of passively evolving galaxies at the two redshifts and by comparing the upper cutoff in their age distributions. All selected galaxies need to have similar metallicities and low star formation rates (i.e., a red color) so that the average age of their stars would far exceed the age difference between the two galaxy samples, $\Delta t$.

This differential age method is much more reliable than a method based on an absolute age determination for galaxies (e.g., Dunlop et al. 1996; Alcaniz \& Lima 2001; Stockton 2001). As demonstrated in the case of globular clusters, absolute stellar ages are more vulnerable to systematic uncertainties than relative ages (Stetson, Vandenberg, \& Bolte 1996). Moreover, absolute galaxy ages can only provide a lower limit to the age of the universe and only place weak constraints on the possible histories of $w_{Q}(z)$.

The quantity measured in our method is directly related to the Hubble parameter,

$$
H(z)=-\frac{1}{(1+z)} \frac{d z}{d t} .
$$

Hence, an application of this method to old elliptical galaxies in the local universe can be used to determine the value of the current Hubble constant, $H_{0}$.

In $\S 2$ we quantify the advantage of the above differential age method relative to the standard luminosity distance method in constraining the history of $w_{Q}(z)$. We then describe the spectroscopic dating technique and apply it to mock galaxy spectra in $\S 3$. In particular, we analyze the dependence of the cosmological constraints on the signalto-noise ratio $(\mathrm{S} / \mathrm{N})$ of the spectroscopic data and the number of observed galaxies. The significance level of the con- 
straints attainable with a single pair of galaxies dictates the number of such pairs required in order to differentiate between various histories of $w_{Q}(z)$. Finally, we summarize our main conclusions in $\S 4$.

\section{OBSERVABLES}

We consider a flat universe composed of matter and dark energy, the latter having an equation of state $p_{Q}=w_{Q}(z) \rho_{Q}$, where $w_{Q}$ may depend on redshift. The Hubble parameter is $H^{2}=H_{0}^{2}\left[\rho_{T}(z) / \rho_{T}(0)\right]$. Here, the subscripts $Q, m$, and $T$ refer to the dark energy, the matter, or the total sum of the two, respectively. Assuming further that the matter is nonrelativistic (i.e., effectively presureless), we get

$$
\begin{aligned}
& H_{0}^{-1} \frac{d z}{d t}=-(1+z) \frac{H(z)}{H_{0}} \\
&=-(1+z)^{5 / 2}\left\{\Omega_{m}(0)+\Omega_{Q}(0)\right. \\
&\left.\quad \times \exp \left[3 \int_{0}^{z} \frac{d z^{\prime}}{\left(1+z^{\prime}\right)} w_{Q}\right]\right\}^{1 / 2},
\end{aligned}
$$

where we have used the energy conservation equation for the dark energy, $\dot{\rho}_{Q}=-3 H\left(1+w_{Q}\right) \rho_{Q}$ (Maor et al. 2001). Thus, $d z / d t$ is related to the equation of state of the dark energy through one integration only, while the luminosity distance in equation (1) is given by an integral of the inverse of equation (3), namely, through two integrations. By differentiating $d z / d t$ with respect to $t$, we find

$$
\begin{aligned}
H_{0}^{-2} \frac{d^{2} z}{d t^{2}}= & \frac{\left[H_{0}^{-1}(d z / d t)\right]^{2}}{(1+z)}\left[\frac{5}{2}+\frac{3}{2} w_{Q}(z)\right] \\
& -\frac{3}{2} \Omega_{m}(0)(1+z)^{4} w_{Q}(z)
\end{aligned}
$$

which depends explicitly on $w_{Q}$ without any integrations. Thus, the second derivative of redshift with respect to cosmic time measures $w_{Q}$ directly. While it is possible to find significantly different redshift histories of $w_{Q}$ for which the evolution of $d_{L}$ is similar, this cannot be done for $d^{2} z / d t^{2}$.

Maor et al. (2001) have argued that because of its integral form, the luminosity distance has only a weak discriminating power with respect to different possible histories of $w_{Q}(z)$. To demonstrate their case, they considered examples of $w_{Q}$, which have a quadratic dependence on $z$. Figure 1 shows similar examples (top panel) along with their corresponding observables, namely, the luminosity distance $d_{L}(z)$ (second panel), $d z / d t$ (fourth panel), and $d^{2} z / d t^{2}$ (sixth panel). In the panel below each of these observables, we show the percentage variations relative to our fiducial case of $w_{Q}=-0.7$. The different $w_{Q}(z)$ histories generate a variation of $\sim 2 \%$ in $d_{L}, \sim 10 \%$ in $d z / d t$, and $\sim 30 \%$ in $d^{2} z / d t^{2}$ at $z \sim 1.5$. The last observable has a fractional variation as large as that of $w_{Q}(z)$.

In order to illustrate more effectively the problem with $d_{L}(z)$ as an indicator of $w_{Q}(z)$, we have chosen (following Maor et al. 2001) cases of $w_{Q}(z)$ that give nearly the same behavior as $d_{L}(z)$. The case with $w_{Q}=-1$ is shown only to illustrate the required ability of $S N A P$ to distinguish our fiducial case with $w_{Q}=-0.7$ from a different value of $w_{Q}$. By inspection of the third panel from the top in Figure 1, it is clear that the different $w_{Q}$ cases lead to $d_{L}$ values that are different by at most $3 \%$ from our fiducial case in the redshift

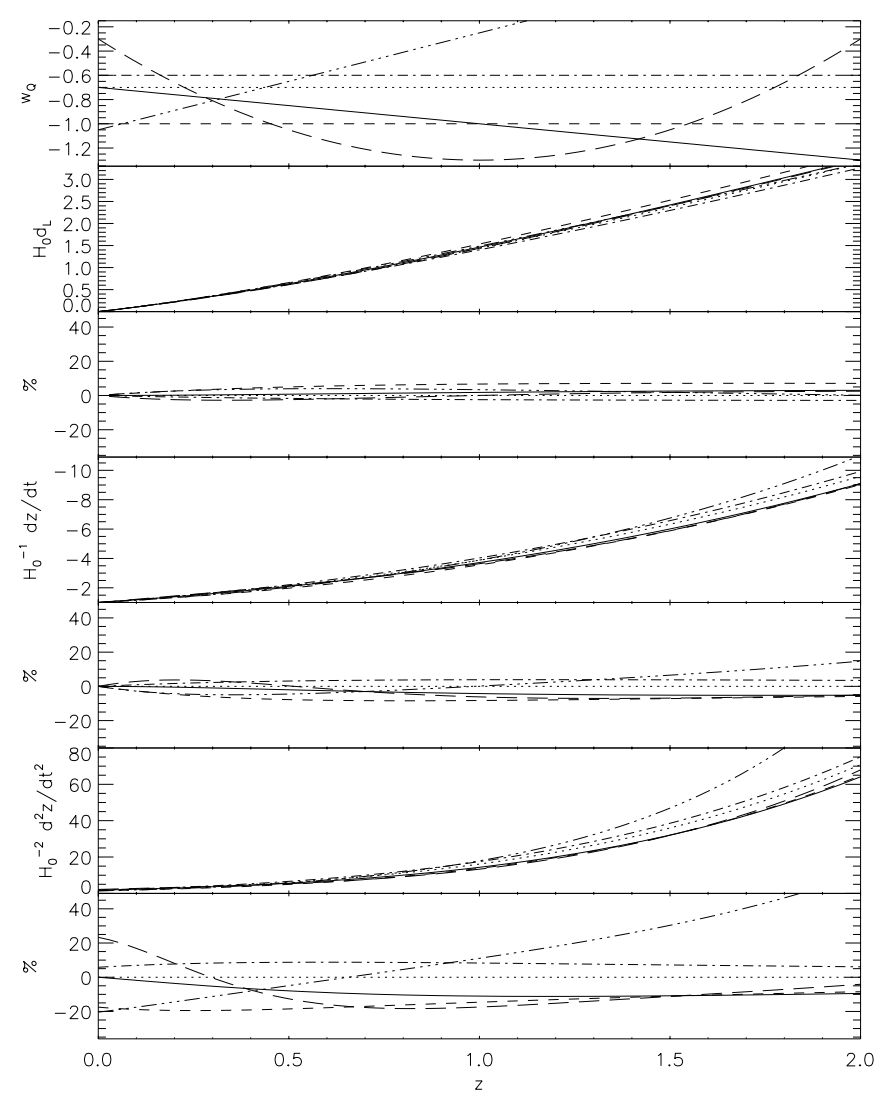

FIG. 1.- Several examples of $w_{Q}(z)$ as a function of redshift (top panel) and the redshift dependence of several observables in these cases. The observables are $d_{L}$ (second panel), $d z / d t$ (fourth panel), and $d^{2} z / d t^{2}$ (sixth panel). In the panel immediately below each of these three panels, we show the corresponding percentage variation of the observables under consideration relative to our fiducial model with $w_{Q}=-0.7$. The third panel from the top shows that variations among different models for $d_{L}$ are not larger than $3 \%$.

range of interest. This is the ultimate detection limit for $S N A P$, which could be achieved under the most optimistic conditions, i.e., assuming that the data follow the theoretical estimations with no additional systematic errors (see Maor et al. 2001). ${ }^{4}$ The fifth and seventh panels from the top show the percentage variation for $d z / d t$ and $d^{2} z / d t^{2}$, respectively. For $d z / d t$ the variation is of about $10 \%$, while for $d^{2} z / d t^{2}$ it is of order $20 \%$. The crucial point is that no form of $w_{Q}(z)$ can make the results degenerate below the $10 \%$ and $20 \%$ levels, respectively, for these two indicators.

\section{SPECTROSCOPIC DATING OF PASSIVELY EVOLVING GALAXIES}

The change of cosmic time with redshift may be inferred from the aging of stellar populations in galaxies. This inference must be done with caution because galaxies are vigorous sites of star formation at high redshift. It might seem difficult to estimate accurately the differential aging of the universe based on star-forming galaxies because the stars in these galaxies are born continuously and a young stellar population may dominate their emission spectrum. Fortunately, examples of passively evolving galaxies have already

\footnotetext{
${ }^{4}$ See also http://snap.lbl.gov.
} 
been identified in large numbers at high redshifts (Dunlop 1996; Daddi, Cimatti, \& Renzini 2000; A. Dey et al. 2002, in preparation; Stockton 2001). We focus our attention on these red galaxies because their light is dominated by an old stellar population.

Our goal is to measure the age difference between two passively evolving galaxies at different redshifts. How accurately can this be done? We emphasize that the relative age is better determined than the absolute age because systematic effects on the absolute scale are factored out (for a fractional age difference $\ll 1)$. In principle, one would like to determine $w_{Q}$ at all redshifts, but it may be easier to first determine where the variation is maximum. Therefore, in the following discussion we have chosen to focus on the redshift interval where the $d z / d t$ indicator varies the most among the different potential histories of $w_{Q}(z)$ (see Fig. 1). In addition, at redshift $1-1.5$ the rest-frame UV redshifts into the optical band to which spectrographs of most $10 \mathrm{~m}$ telescopes are sensitive, and this makes it easier to date the observed galaxies. Note, however, that $d^{2} z / d t^{2}$ is able to track the $w_{Q}$ variation, and therefore one can measure $w_{Q}$ at redshift zero where observing conditions are much easier, although at low redshift the UV part of the spectrum, which greatly constrains the age, will have to be observed from space.

At redshifts $z \sim 1-2$, the rest-frame UV spectrum of nonstar-forming (elliptical) galaxies is dominated by light from main-sequence stars with masses of $1-2 M_{\odot}$ in a regime of stellar evolution that is well understood (Spinrad et al. 1997). The lack of interstellar dust or gas simplifies further the spectrum of old elliptical galaxies. Furthermore, because of the narrow mass range of stars dominating the UV light, $\Delta M$ less than $0.3 M_{\odot}$, variations of the initial mass function play no role in our age determination (see discussion in Spinrad et al. 1997). The top panel in Figure 2 shows a simulated galaxy spectrum in the rest-frame UV that can be easily collected by $10 \mathrm{~m}$ class telescopes from the ground (e.g., Dunlop et al. 1996). This spectrum was calculated for a single stellar population with a specific metallicity (solar) and age (2.4 Gyr) using the synthetic stellar population models developed in Jimenez et al. (1998). The simulated spectrum has a $10 \AA$ resolution, to which we have added Poisson photon noise with $\mathrm{S} / \mathrm{N}=30$ per resolution element. The bottom panel shows the values recovered at the $95 \%$ confidence level (using a $\chi^{2}$ goodness-of-fit statistics) for the metallicity and age with $\mathrm{S} / \mathrm{N}=30$ (dark shaded region) and $\mathrm{S} / \mathrm{N}=50$ (lightly shaded inner region). Note that age and metallicity are not degenerate in the rest-frame UV, provided that the measured spectra have a sufficient $\mathrm{S} / \mathrm{N}$ (Nolan et al. 2001). Also, a wider spectral coverage helps in breaking the age-metallicity degeneracy. A value of $\mathrm{S} / \mathrm{N}=15-30$ can be obtained for a faint galaxy of magnitude 24 in the $R$ band after $40 \mathrm{hr}$ of integration on the Keck telescope (K. Adelberger 2002, private communication). ${ }^{5}$ This estimate is for the best observing conditions and instrumentation in the above telescopes. Observation of a sufficiently wide field of view would allow us to measure spectra of many passively evolving galaxies at the same time. This can help to offset the costly effort of obtaining very high $\mathrm{S} / \mathrm{N}$ spectra.

\footnotetext{
${ }^{5}$ See also http://www.gemini.edu/sciops/instruments/gmos/
}
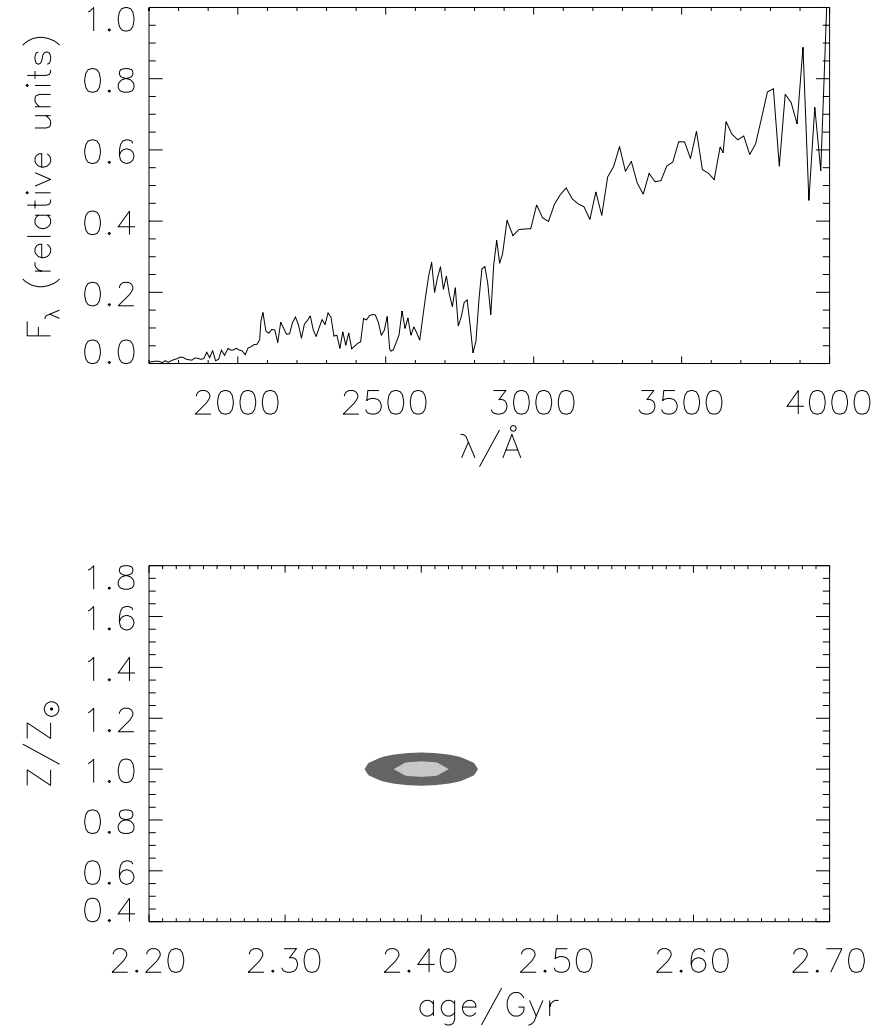

FIG. 2.-Top panel shows a simulated spectrum (flux per unit wavelength, $F_{\lambda}$ ) for a galaxy with a solar metallicity and an age of $2.4 \mathrm{Gyr}$, including Poisson photon noise with $\mathrm{S} / \mathrm{N}=30$ at a spectral resolution of $10 \AA$. The bottom panel shows the accuracy of the metallicity and age recovery for $\mathrm{S} / \mathrm{N}=30$ (dark shaded region) and $\mathrm{S} / \mathrm{N}=50$ (light shaded region)

The assumption that stars in an elliptical galaxy have a single metallicity value is clearly an oversimplification. Local ellipticals have metallicity gradients (e.g., Davies et al. 1993), even though these gradients are mild (e.g., Friaca $\&$ Terlevich 2001). We therefore consider the possibility that elliptical galaxies have a mixture of populations with different metallicities. ${ }^{6}$ In order to assess the accuracy by which we can recover the age of a mixed stellar population, we simulated several spectra with a mixture of six metallicity values ranging from 0.01 to 5 times the solar value. For example, one of our models had weights of $5 \%, 10 \%, 25 \%$, $20 \%, 12 \%$, and $28 \%$ for metallicities of $0.01,0.2,1,2.5$, and 5 times the solar value, respectively. We then attempted to recover the age by searching for the best-fit model with a single metallicity and found an age uncertainty of $0.12 \mathrm{Gyr}$ for our fiducial model with an age of $2.4 \mathrm{Gyr}$ (and an uncertainty of $\sim 0.8 \mathrm{Gyr}$ for an age of $15 \mathrm{Gyr}$ ); i.e., the fractional age uncertainty is $\sim 5 \%$. Alternatively, we fitted the simulated spectrum by a mixed metallicity model with six free components and recovered a more accurate age with an uncertainty of $\sim 0.05 \mathrm{Gyr}$ (as well as a reduced metallicity uncertainty of $8 \%$ ).

\footnotetext{
${ }^{6}$ The small spread in age among stars in an elliptical galaxy does not affect our results because the method relies only on the shift in the average stellar age as a function of redshift. For a passively evolving galaxy, the shift in the average age of its stars within a given redshift interval is determined only by the background cosmology.
} 


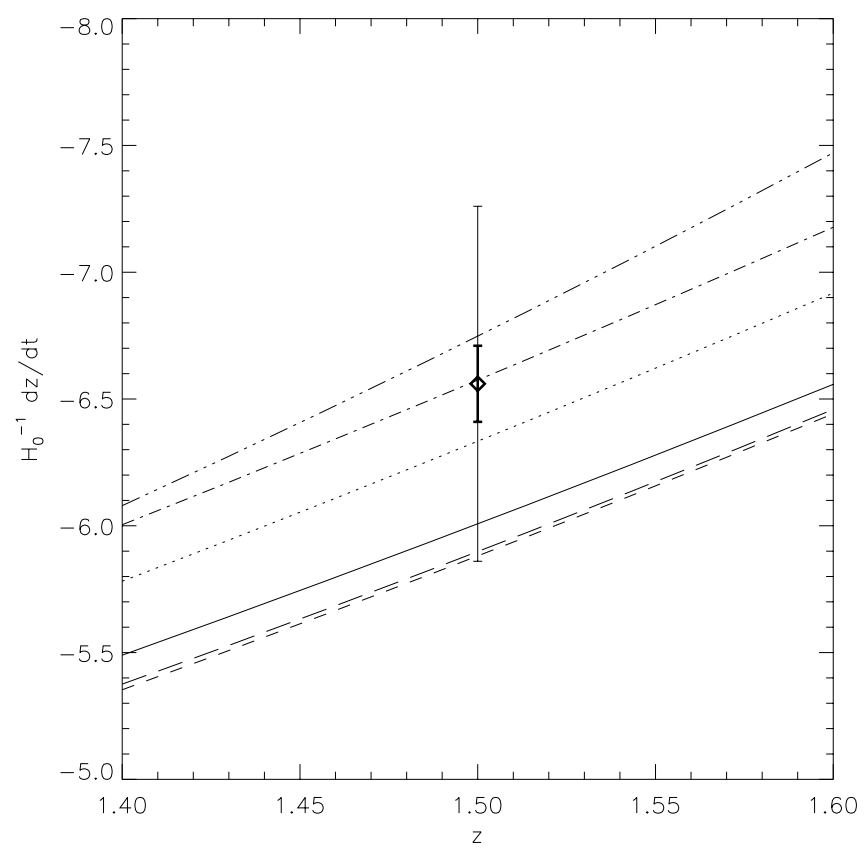

FIG. 3.-Large error bar illustrates the constraint that can be placed on $w_{Q}(z)$ by using only a single pair of red galaxies that formed at the same time and are observed at $z=1.6$ and $z=1.4$. The spectra are assumed to have a spectral resolution of $10 \AA$ and $\mathrm{S} / \mathrm{N}=30$. When data for 20 such pairs are available, the uncertainty is reduced to the thicker, smaller error bar.

Using the less optimistic age uncertainty of 5\% derived above for $\mathrm{S} / \mathrm{N}=30$, we may now estimate the attainable constraints on $w_{Q}(z)$ for a fiducial pair of passively evolving galaxies that formed at the same time but are observed at $z=1.4$ and 1.6 and have ages of 2.79 and $2.4 \mathrm{Gyr}$, respectively. Figure 3 shows the resulting error bar on $d z / d t$, which is not sufficiently restrictive for a single pair of galaxies. However, for a statistical ensemble of independent galaxies (which do not all belong to the same local region of a galaxy cluster, ${ }^{7}$ for example), the error bar will be reduced by the square root of the number of analyzed galaxy pairs. The smaller error bar in the plot illustrates the constraint that could be placed by 20 such pairs. This constraint is sufficient to distinguish among the different $w_{Q}(z)$ histories in Figure 1.

The situation in reality is not as simple as described above because a galaxy survey will not just identify galaxies that formed at the same cosmic time. Rather, the galaxies selected as not having substantial star formation will show an age distribution at any given redshift. Given this age distribution, what is the accuracy by which one may recover the age shift, provided that the age of each passively evolving galaxy is measured with a 5\% uncertainty? To answer this question, we have simulated the galaxy age distributions at two different redshifts. The distribution at the higher redshift was modeled fiducially as a Schechter function, which is

\footnotetext{
${ }^{7}$ We have excluded cluster elliptical galaxies from this test because they potentially introduce a systematic bias. The galaxies that belong to the overdense region of a cluster might not be representative of the rest of the universe. In comparing clusters at different redshifts, one must be sure that the clusters have similar general properties (e.g., belong to the same richness class) and that differences between the clusters that are being compared do not introduce environmental biases.
}

asymptotically flat at low ages and is fully truncated at some finite maximum age value (because galaxies cannot be older than the age of the universe). In our example we chose a cutoff at 2.4 Gyr. The distribution was then binned into bins of 0.1 Gyr width, corresponding to the age uncertainty for individual galaxies. The age distribution at a lower redshift was chosen to be the same as the first one but shifted by either $0.0,0.15$, or $0.30 \mathrm{Gyr}$ (so that the maximum age in the distribution would be $2.4,2.55$, or $2.7 \mathrm{Gyr}$, respectively). We also removed or added a fixed fraction (20\%) of the galaxies within each bin through a random Poisson realization of this fraction. This degree of freedom is required because some galaxies may have merged with a star-forming galaxy and therefore disappeared from the old galaxy population. Moreover, the normalization of the two distributions is regarded as a free parameter because the volume surveyed at the two redshifts might be different. Our approach assumes that both samples of galaxies have been passively evolving for a time much longer than the age difference between the two redshifts. Each member of the low-redshift sample has a progenitor, which, statistically speaking, is included in the high-redshift sample. But each high-redshift galaxy has some probability of encountering a merger and disappearing from the low-redshift sample. Our method would fail only if this probability is both significantly large and significantly age-dependent. However, for a sufficiently narrow redshift interval, the merger probability is small. The tight color-magnitude relation of present-day elliptical galaxies further limits the effect of mergers on their age distribution (Peacock 1991; Bower, Lucey, \& Ellis 1992; Peacock et al. 1998). To be more quantitative, because in a hierarchy galaxy formation takes place through the buildup of smaller units, some stars will be formed before the collapse of the elliptical galaxy. This means that the derived age for the galaxy will be age $=t\left(z_{\text {obs }}\right)-t\left(z_{c}\right)+\alpha t\left(z_{c}\right)$. Constraints imposed by the tightness of the colormagnitude relation imply that $\alpha \approx 0.3$. This translates into the number of mergers that are expected in a given redshift interval. As an example, consider an elliptical galaxy observed at $z=1.5$; what higher redshift does one need to consider in order to be sure there was no merger? From the above formula, this occurs when the age difference between the two redshifts is equal to $0.3 t\left(z_{c}\right)$, where $t\left(z_{c}\right)$ is the age of the universe when the higher redshift galaxy collapses. For an Einstein-de Sitter universe this will correspond to $z \approx 1.7$. So a redshift width of 0.2 guarantees that no merger took place.

The two distributions are shown as the two top panels of Figure 4 for an age shift of 0.3 Gyr and a total of $\sim 75$ galaxies in each distribution. We binned the distributions for illustrative purposes. The best statistical method to recover the shift is the Kolmogorov-Smirnov (K-S) test on the unbinned data. The bottom panel shows the probability for getting a particular value of the peak in the K-S probability (Press et al. 1992) for the three cases we consider. We derived this probability distribution through a large number of Monte Carlo realizations of the above distributions. Also shown as shaded regions are the shift levels for which one could distinguish among the different $w_{Q}(z)$ histories in Figure 3 . We find that the shifts can be recovered with good precision, despite the fact that the tip of the age distribution is populated by a small number of galaxies. In particular, we get a $\gtrsim 90 \%$ probability in this example of distinguishing among the different $w_{Q}(z)$ histories. We have experimented 

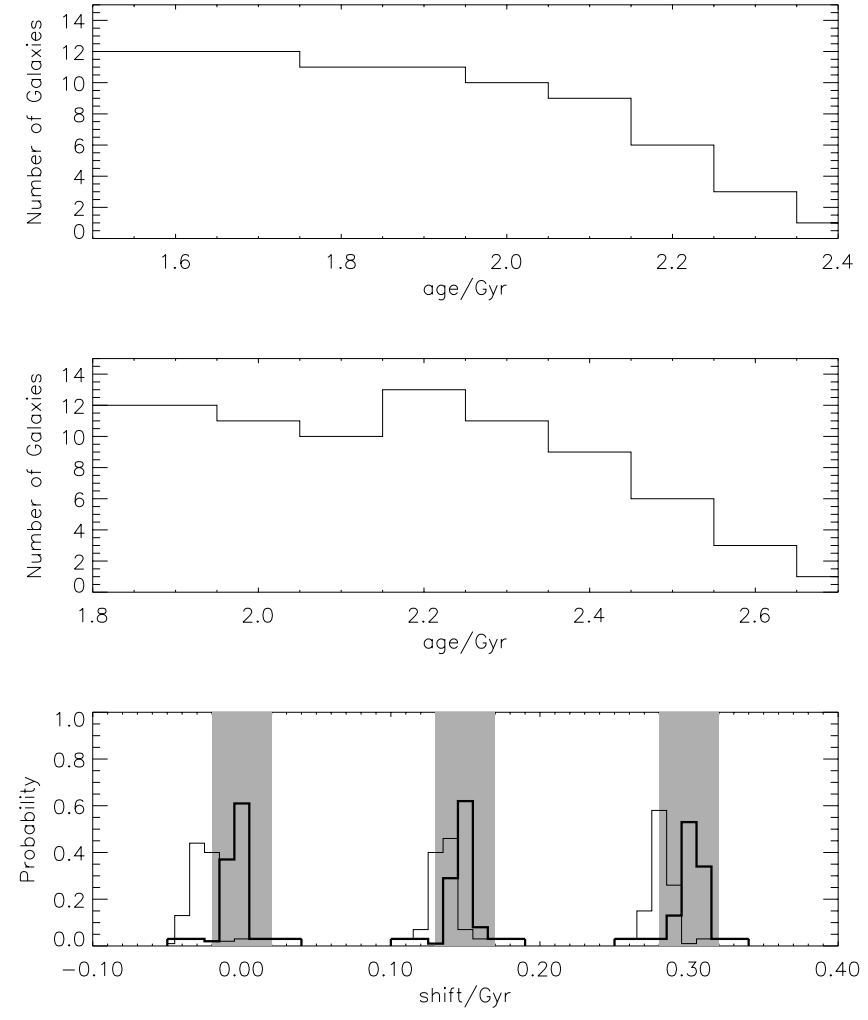

FIG. 4.-Top panel shows a hypothetical age distribution for 75 galaxies at a redshift $z=1.6$. The middle panel shows the same distribution at $z=1.4$, in which all galaxies are older by $0.3 \mathrm{Gyr}$ and their fraction per bin changes by $\pm 20 \%$ with Poisson fluctuations (resulting from mergers). The bottom panel shows the probability distribution of the recovered shift in age between the two galaxy distributions using the peak probability value of the K-S likelihood test for unbinned data with an age error of $0.1 \mathrm{Gyr}$ galaxy $^{-1}$. The thin line corresponds to the case where bins with age greater than $2.5 \mathrm{Gyr}$ from the middle panel have been removed. We show three cases of shifts, namely, $0.0,0.15$, and $0.3 \mathrm{Gyr}$. The shaded regions show the range in the recovered shift that would allow us to separate the different $w_{Q}(z)$ histories in Fig. 3.

with removing the oldest bins (ages greater than 2.5 in the middle panel of Fig. 4) in the second redshift interval. We find that the shift in age can still be recovered with the needed accuracy. This is shown as a thin line in the bottom panel of Figure 4. The reason for this is because the histogram has a curvature and therefore it is not possible to simply rescale the number of galaxies down by some factor and recover a zero shift instead of $0.3 \mathrm{Gyr}$. Of course, the accuracy in the recovered shift is not as good as when the old bins are present. If the histograms were pure power laws, then the shift determination would be compromised by the freedom to rescale their vertical axis.

At present, there is no such set of galaxy spectra that could discriminate between a constant and a variable $w_{Q}(z)$. Nevertheless, it is instructive to examine the best spectroscopically observed pair of red galaxies to date, namely, 53W091 and 53W069, which reside at $z=1.43$ and 1.55, respectively. The stellar populations of these galaxies have been studied in detail recently by Nolan et al. (2001), who demonstrated that the age-metallicity degeneracy can be broken based on UV rest-frame spectroscopy with high quality. However, because these galaxies were observed with only $\mathrm{S} / \mathrm{N} \sim 6-10$, they do not provide strong constraints on $w_{Q}(z)$.

\section{CONCLUSIONS}

We have shown that the derivative of cosmic time with respect to redshift can be used as an effective tool for measuring the equation of state of the dark energy as a function of redshift. For this purpose, spectra of passively evolving galaxies need to be obtained with a high $\mathrm{S} / \mathrm{N}$. In order to differentiate at the $2 \sigma$ level between a constant and a variable $w_{Q}(z)$, one needs $\sim 70$ pairs of galaxies at $R \sim 24$. For a field of view $\left(\sim 10 \mathrm{arcmin}^{2}\right)$, which is attainable with instruments like DEIMOS on the Keck telescope and VIMOS on the Very Large Telescope, we estimate that the required number of ellipticals may be found in the volume between $z=1.4$ and 1.6, and so all pairs can be observed simultaneously over $40 \mathrm{hr}$ of integration on a $10 \mathrm{~m}$ class telescope, such as Keck or the VLT.

The key advantages of our method relative to supernova searches such as the SNAP space mission are that (1) the observable $d z / d t$ is more sensitive to $w_{Q}(z)$ than the luminosity distance $d_{L}$ and (2) the related precision observations can be done from the ground. In fact, some observational groups have already identified relevant sets of red galaxies at high redshifts (e.g., Daddi et al. 2000; Stockton 2001).

In our discussion we have assumed that $\Omega_{m}(0)$ will already be known by the time this analysis is done, based on forthcoming high-precision data from the Microwave Anisotrophy Probe satellite $(M A P)^{8}$ and the Sloan Digital Sky Survey (SDSS). ${ }^{9}$ Preliminary attempts to determine $\Omega_{m}(0)$ by combining microwave background and large-scale structure data appear promising (see, e.g., Efstathiou et al. 2002). We note that the value of $\Omega_{m}(0)$ adds only a single constant parameter to our analysis and should not compromise significantly the measurement of the function $w_{Q}(z)$, as long as $d z / d t$ is measured at many different redshifts.

The Hubble constant, $H_{0}$, can be measured by computing the age difference between red galaxies at $z=0$ and $z \sim 0.2$. The characteristic age difference in this redshift range, $\sim 2$ Gyr, can be measured for a single pair of galaxies with a precision of $\sim 20 \%$ given data with $\mathrm{S} / \mathrm{N}=10$ and spectral resolution of $10 \AA$. A large volume of spectroscopic data with this quality on red galaxies in the local universe will soon be released by SDSS. The wavelength coverage of the SDSS spectra of 3900-9100 A includes spectroscopic features such as $\mathrm{H} \beta$ that are sensitive to the aging of the stellar population. Given a statistical sample of many hundreds of red SDSS galaxies (D. Eisenstein 2002, private communication), one could in principle determine the value of $H_{0}$ to a percent accuracy, as long as the systematic modeling uncertainties for relative ages can be reduced to that level. The differential age method is independent of the Cepheid distance scale and is subject to other uncertainties.

The quantity $d^{2} z / d t^{2}$ tracks directly $w_{Q}$ (see bottom panel of Fig. 1). Because the variation of $w_{Q}$ can be quite pronounced at low redshifts, a measurement of $d^{2} z / d t^{2}$ from a large sample of passively evolving galaxies, like red SDSS galaxies, will lead to a direct determination of $w_{Q}$. This measurement can be further improved if resolved globular clusters are observed in nearby galaxies and their colormagnitude diagram can be constructed down to the main-sequence turnoff, as will be the case with the Next Generation Space Telescope.

\footnotetext{
${ }^{8} M A P$ satellite data are available at http://map.gsfc.nasa.gov.

${ }^{9}$ SDSS data are available at http://www.sdss.org.
} 
We thank Kurt Adelberger for useful discussions, Rennan Barkana for helpful comments on the manuscript, and Rob Caldwell and Marc Kamionkowski for useful discus- sions on ages and the equation of state. This work was supported in part by NASA grants NAG 5-7039 and NAG 5-7768 and by NSF grants AST-9900877 and AST-0071019.
Alcaniz, J. S., \& Lima, J. A. S. 2001, ApJ, 550, L133

Bower, R. G., Lucey, J. R., \& Ellis, R. S. 1992, MNRAS, 254, 601

Caldwell, R. R., Dave, R., \& Steinhardt, P. J. 1998, Ap\&SS, 261, 303

Daddi, E., Cimatti, A., \& Renzini, A. 2000, A\&A, 362, L45

Davies, R. L., Sadler, E. M., \& Peletier, R. F. 1993, MNRAS, 262, 650

de Bernardis, P., et al. 2000, Nature, 404, 955

Dunlop, J., Peacock, J., Spinrad, H., Dey, A., Jimenez, R., Stern, D., \&

Windhorst, R. 1996, Nature, 381, 581

Efstathiou, G., et al. 2002, MNRAS, 330, L29

Freedman, W. L., et al. 2001, ApJ, 553, 47

Friaca, A. C. S., \& Terlevich, R. J. 2001, MNRAS, 325, 335

Garnavich, P. M., et al. 1998, ApJ, 509, 74

Hanany, S., et al. 2000, ApJ, 545, L5

Huterer, D., \& Turner, M. S. 2001, Phys. Rev. D, 64, 123527

Jimenez, R., Padoan, P., Matteucci, F., \& Heavens, A. F. 1998, MNRAS, 299,123

Lee, A. T., et al. 2001, ApJ, 561, L1

Maor, I., Brustein, R., \& Steinhardt, P. J. 2001, Phys. Rev. Lett., 86, 6

Netterfield, C. B., et al. 2002, ApJ, in press (astro-ph/0104460)

\section{REFERENCES}

Nolan, L. A., Dunlop, J. S., Jimenez, R., \& Heavens, A. F. 2001, MNRAS, submitted (astro-ph/0103450)

Peacock, J. A. 1991, in Proc. 2nd Rencontres de Blois, Physical Cosmology, ed. A. Blanchard, L. Celnekier, M. Lachieze-Rey, \& J. Tran Thanh Van (Gif-sur-Yvette Cedex: Editions Frontières), 337

Peacock, J. A., et al. 1998, MNRAS, 296, 1089 .2001, Nature, 410, 169

Perlmutter, S., et al. 1999, ApJ, 517, 565

Press, W. H., Teukolsky, S. A., Vetterling, W. T., \& Flannery, B. P. 1992, Numerical Recipes (Cambridge Univ. Press)

Ratra, B., \& Peebles, P. J. E. 1988, Phys. Rev. D, 37, 3406

Riess, A. G., et al. 1998, AJ, 116, 1009

Spinrad, H., et al. 1997, ApJ, 484, 581

Stetson, P. B., Vandenberg, D. A., \& Bolte, M. 1996, PASP, 108, 560

Stockton, A. 2001, in ASP Conf. Ser. 245, Astrophysical Ages and Time Scales, ed. T. von Hippel, C. Simpson, \& N. Manset (San Francisco: ASP), 517

Weller, J., \& Albrecht, A. 2001, Phys. Rev. Lett., 86, 1939 